\title{
Machine learning for screening prioritization in systematic reviews: comparative performance of Abstrackr and EPPI-Reviewer
}

Amy Y. Tsou*, Jonathan R. Treadwell, Eileen Erinoff and Karen Schoelles

\begin{abstract}
Background: Improving the speed of systematic review (SR) development is key to supporting evidence-based medicine. Machine learning tools which semi-automate citation screening might improve efficiency. Few studies have assessed use of screening prioritization functionality or compared two tools head to head. In this project, we compared performance of two machine-learning tools for potential use in citation screening.

Methods: Using 9 evidence reports previously completed by the ECRI Institute Evidence-based Practice Center team, we compared performance of Abstrackr and EPPI-Reviewer, two off-the-shelf citations screening tools, for identifying relevant citations. Screening prioritization functionality was tested for 3 large reports and 6 small reports on a range of clinical topics. Large report topics were imaging for pancreatic cancer, indoor allergen reduction, and inguinal hernia repair. We trained Abstrackr and EPPI-Reviewer and screened all citations in 10\% increments. In Task 1, we inputted whether an abstract was ordered for full-text screening; in Task 2, we inputted whether an abstract was included in the final report. For both tasks, screening continued until all studies ordered and included for the actual reports were identified. We assessed potential reductions in hypothetical screening burden (proportion of citations screened to identify all included studies) offered by each tool for all 9 reports.
\end{abstract}

Results: For the 3 large reports, both EPPI-Reviewer and Abstrackr performed well with potential reductions in screening burden of 4 to 49\% (Abstrackr) and 9 to 60\% (EPPI-Reviewer). Both tools had markedly poorer performance for 1 large report (inguinal hernia), possibly due to its heterogeneous key questions. Based on McNemar's test for paired proportions in the 3 large reports, EPPI-Reviewer outperformed Abstrackr for identifying articles ordered for full-text review, but Abstrackr performed better in 2 of 3 reports for identifying articles included in the final report. For small reports, both tools provided benefits but EPPI-Reviewer generally outperformed Abstrackr in both tasks, although these results were often not statistically significant.

Conclusions: Abstrackr and EPPI-Reviewer performed well, but prioritization accuracy varied greatly across reports. Our work suggests screening prioritization functionality is a promising modality offering efficiency gains without giving up human involvement in the screening process.

Keywords: Machine learning, Citation screening, Text-mining, Abstrackr, EPPI-Reviewer, Screening prioritization, Methodology, Screening burden, Efficiency

\footnotetext{
* Correspondence: atsou@ecri.org

Center for Clinical Excellence and Guidelines, ECRI Institute, Evidence-based

Practice Center, 5200, Plymouth Meeting, PA 19462-1298, USA
}

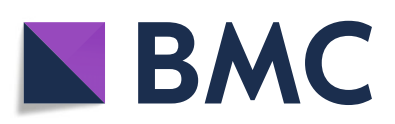

(c) The Author(s). 2020 Open Access This article is licensed under a Creative Commons Attribution 4.0 International License, which permits use, sharing, adaptation, distribution and reproduction in any medium or format, as long as you give appropriate credit to the original author(s) and the source, provide a link to the Creative Commons licence, and indicate if changes were made. The images or other third party material in this article are included in the article's Creative Commons licence, unless indicated otherwise in a credit line to the material. If material is not included in the article's Creative Commons licence and your intended use is not permitted by statutory regulation or exceeds the permitted use, you will need to obtain permission directly from the copyright holder. To view a copy of this licence, visit http://creativecommons.org/licenses/by/4.0/ The Creative Commons Public Domain Dedication waiver (http://creativecommons.org/publicdomain/zero/1.0/) applies to the data made available in this article, unless otherwise stated in a credit line to the data. 


\section{Background}

Systematic reviews (SRs) play a vital role across many disciplines including education, criminal justice, and particularly healthcare, enabling evidence-based medicine by synthesizing and critically appraising bodies of literature to produce a comprehensive and critical assessment of what is known [1,2]. In particular, SRs impact patient care as the basis for development of high-quality clinical practice guidelines $[3,4]$. Despite their importance, creating SRs in a timely fashion remains challenging due to the lengthy process required for development, which typically requires months to years [5].

One particularly time-consuming step in developing SRs is citation screening. Typically, thousands of abstracts are reviewed to identify only a small number of relevant studies. While laborious, this process reflects the priority systematic reviewers place on identifying all relevant studies to avoid bias (i.e., attaining 100\% sensitivity) [6]. Wallace et al. estimated that for an experienced reviewer, screening 5000 abstracts requires $40 \mathrm{~h}$ of uninterrupted work for simple topics, with significantly more time for complex topics [7]. In one project at our institution consisting of five large SRs (for use in guideline development), searches identified a combined 31, 608 citations, for which we screened 13,492 abstracts in $297 \mathrm{~h}$, or 7.4 full weeks for one investigator.

Semi-automating this process using innovative citation screening tools represents one potential solution $[8,9]$. Using machine-learning algorithms trained on a subset of citations, these tools order citations for screening, presenting abstracts with the highest probability of meeting inclusion criteria first (see Fig. 1). This process, known as screening prioritization does not directly reduce the number of abstracts which require screening. However, by presenting reviewers with citations more likely to be included, screening prioritization can improve efficiency by promoting early identification of relevant citations, accelerating retrieval of full-text articles and data extraction and workflow planning $[10,11]$.

Screening prioritization can also support another strategy for improving efficiency, screening truncation, in which citations that fall beneath a specified probability for inclusion are automatically excluded (e.g., < $0.1 \%$ ). This functionality is sometimes described as automatic classification, since the machine "classifies" the remaining unscreened abstracts as so unlikely to be relevant that they do not require screening and can simply be designated as "excluded" [10]. Thus, unlike screening prioritization, screening truncation can allow reviewers to screen fewer abstracts.

Together, screening prioritization and screening truncation can decrease screening burden, which may be defined as the proportion of citations screened in order to identify all relevant studies. Citation screening tools utilizing these functionalities have the potential to reduce workload by 30 to $70 \%$ (with estimates as high as 90\%) [12-14]. However, because truncating the screening process risks missing important studies, this strategy has not been widely adopted [10]. Assessing the accuracy of screening prioritization (to determine to what extent tools can actually identify relevant citations earlier) could help alleviate this concern by identifying various thresholds at which screening truncation may be "safely" performed to avoid missing any relevant studies.

$\square$ Study that should be included

$\square$ Study that should be excluded

BEFORE TRAINING: The includes are randomly dispersed

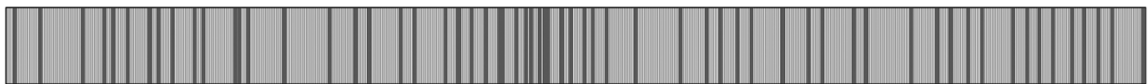

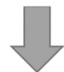

AFTER TRAINING: The remaining studies are ordered by likelihood of inclusion

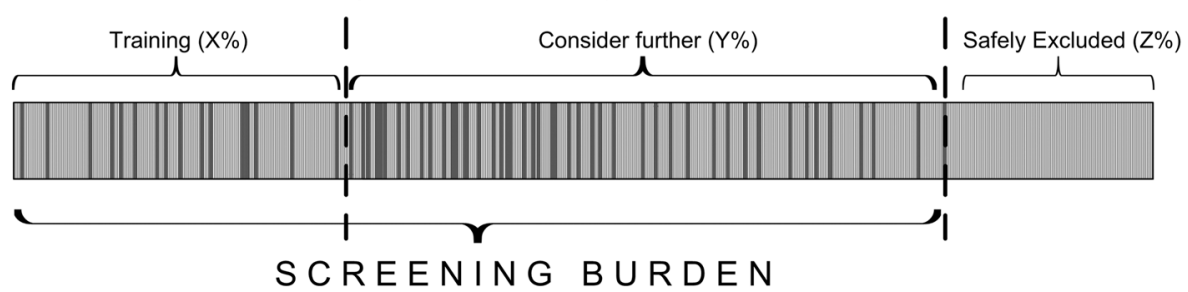

Fig. 1 Screening prioritization and potential reduced screening burden. This figure demonstrates graphically how screening prioritization works. Prior to screening, the articles ultimately included are randomly dispersed (top half). Reviewers train the algorithm by manually including/ excluding studies until a pre-specified number of studies are included. The algorithm then generalizes rules and prioritizes the remaining studies for evaluation by the reviewer 
A 2016 AHRQ Evidence-based practice center (EPC) report surveying the landscape of text-mining tools for use in SR development noted several research priorities including (1) further validation of workload reductions, (2) characterization of potential performance differences for large vs. small datasets, and (3) direct comparisons of tools [15]. To address these research gaps, in this study, we assessed the efficacy of 2 off-the-shelf citation screening tools (Abstrackr and EPPI-Reviewer) to reduce screening burden using screening prioritization. In addition, we also sought to determine if reductions in screening burden differed for large compared to small evidence reports.

\section{Tools of interest: Abstrackr and EPPI-Reviewer}

Abstrackr and EPPI-Reviewer are two off-the-shelf screening tools designed by established systematic review groups. Abstrackr [16, 17] is an open source application currently maintained by the Brown Center for Evidence Synthesis in Health; EPPI-Reviewer [18] was developed at the University College London and is currently used to support evidence synthesis for organizations including the Cochrane Review Groups [14]. Both tools are web-based and facilitate citation screening through text-mining tools and machine learning techniques; they employ "active learning," in which ongoing feedback from reviewers is used dynamically to improve predictive accuracy [14, 17]. Several studies have incorporated Abstrackr into workflow (e.g., as a second screener) and reported a range of improvements in efficiency [19]. For instance, three studies using Abstrackr for screening citations for new reviews (i.e., not updates) reported significant, but variable workload reductions ranging from 9 to $57 \%[7,13,17]$. However, to our knowledge, no studies have assessed EPPI-Reviewer.

\section{Methods}

\section{Study design}

We compared the accuracy of screening prioritization by Abstrackr vs. EPPI-Reviewer using citations from a sample of 9 previously completed evidence reports. We selected 3 evidence reports which had each required screening a large number of citations along with 6 reports which had required screening for a smaller number of citations. Throughout the rest of this report, we describe these reports as "large" or "small" evidence reports.

To be considered for selection, evidence reviews had to assess well-defined medical interventions, be performed since 2010, and screen a minimum number of citations (> 1000 citations for large reports, 200-999 citations for small). For large reviews, we selected 3 AHRQ Evidencebased Practice Center (EPC) reports published from 2012 to 2018; as typical for EPC reports, we performed dual screening. For smaller reports, we selected most from
"Emerging Technology" reports ECRI Institute produces in response to requests from health systems regarding specific interventions. These reports employ standard systematic review methodology (comprehensive search, screening, risk of bias assessment, and strength of evidence appraisal); however, citation screening is performed by only a single analyst.

\section{Citation screening process}

The citation screening process we used for this project is summarized in Fig. 2. While both tools support screening prioritization, there are key differences. Abstrackr updates prediction algorithms once a day (overnight). Conversely, for EPPI-Reviewer, the prediction algorithm updates iteratively (e.g., every 25 citations). With this difference in mind, for each evidence report, we trained each tool using the same sets of citations but different approaches: for Abstrackr, we used a random 10\% sample of all citations; for EPPI-Reviewer, we screened 10 random citations and then engaged the priority screening mode until $10 \%$ of total citations had been screened. Screening continued in 10\% increments. After generation of a prediction model, each tool presented citations in order from highest to lowest likelihood of meeting inclusion criteria.

Systematic reviews may be performed de novo or serve to update a prior existing review. Thus, for each evidence report, we characterized screening burden in two ways (see Table 1). In Task 1, to mimic workflow for a new review, we compared screening performance to decisions made by human reviewers (e.g., should this abstract be included for full-text review). In this scenario, the algorithm could potentially be misled by reviewers choosing to include studies which are ultimately excluded. However, for a review update, an algorithm could be trained solely using known included studies from a prior review (which could dramatically improve accuracy of screening prioritization). To approximate this scenario, in Task 2, we considered whether accuracy of predictions improved when tools were trained using citations ultimately included in the final report.

We exported ordered lists at each $10 \%$ interval (including the percentile rank of the final ordered/included study). Although Abstrackr does provide numerical probabilities of inclusion, we only used the ordered lists of citations to enable comparisons across both tools (as EPPI-Reviewer does not provide probabilities). Screening continued until all ordered/included studies had been screened. For small reports (with fewer citations), we anticipated that Abstrackr or EPPI-Reviewer might have insufficient information to generate a prediction model; in those cases, we planned to continue screening in 10\% increments until all citations had been screened. 


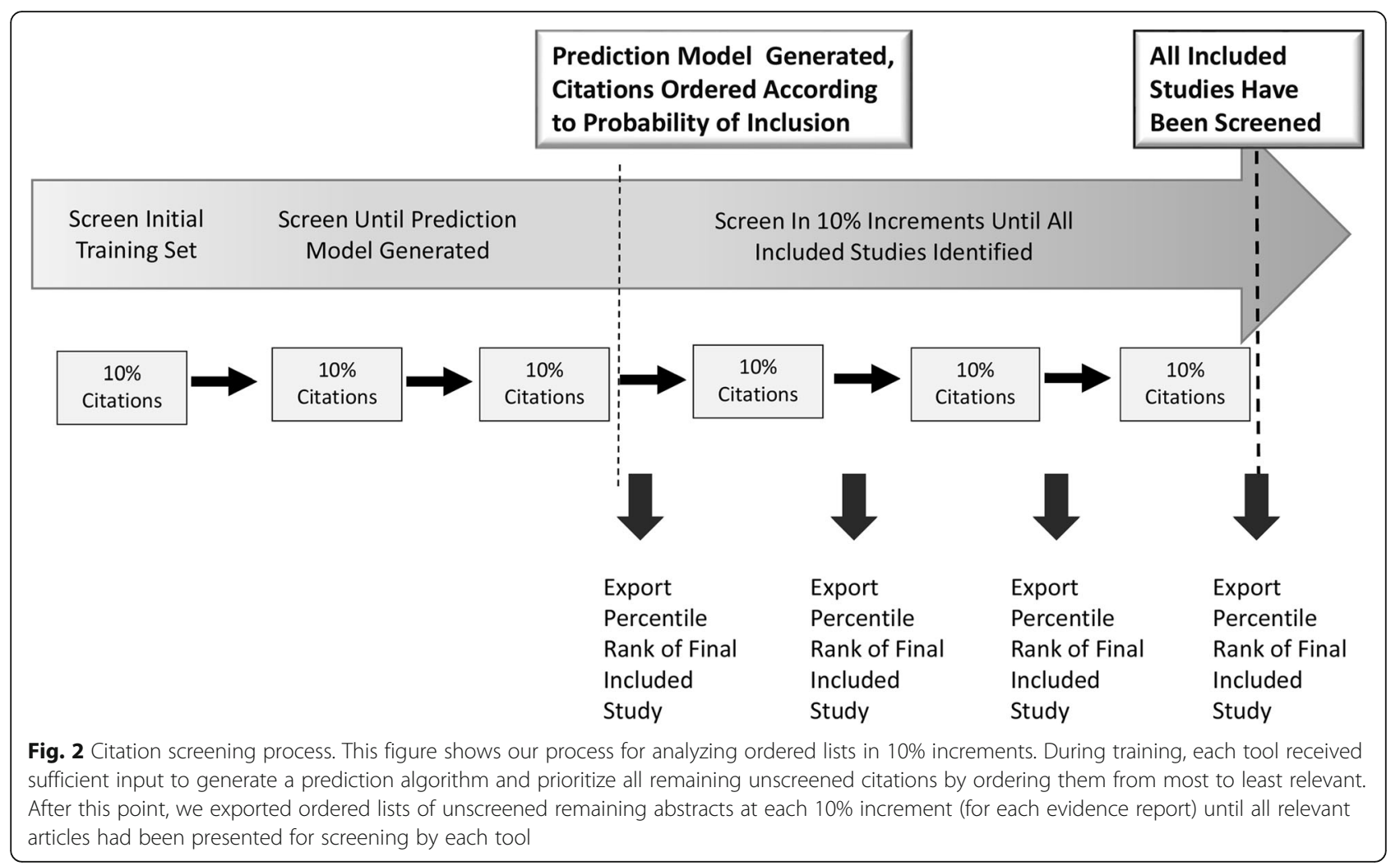

\section{Outcome measures}

Our primary outcome measures were prioritization accuracy and the proportion of included studies identified at various thresholds.

\section{Prioritization accuracy}

For each evidence report, we used the percentile rank of the final included study to determine prioritization accuracy/based on exported predictions, we determined whether each excluded abstract fell within the "screening burden," (the proportion of citations which require screening in order to detect all included studies) for EPPI-Reviewer and Abstrackr respectively. Any particular excluded abstract might fall into the screening burden for neither or both EPPI-Reviewer and Abstrackr; alternatively, excluded abstracts might fall in one tool's screening burden but not the other's. Thus, the data are

Table 1 Task 1 and 2: inputs and outcomes

\begin{tabular}{lll}
\hline & $\begin{array}{l}\text { Input to Abstrackr } \\
\text { /EPPI-Reviewer }\end{array}$ & $\begin{array}{l}\text { Outcome (prioritization } \\
\text { accuracy) definition }\end{array}$ \\
\hline Task 1 & $\begin{array}{l}\text { Did human reviewer(s) } \\
\text { order the study for } \\
\text { full-text review? }\end{array}$ & $\begin{array}{l}\text { Proportion of citations } \\
\text { screened to reach all } \\
\text { citations ordered for } \\
\text { full-text review }\end{array}$ \\
Task 2 & $\begin{array}{l}\text { Was the citation } \\
\text { included in the } \\
\text { final report? }\end{array}$ & $\begin{array}{l}\text { Proportion of citations } \\
\text { screened to reach all } \\
\text { citations included in } \\
\text { the final report }\end{array}$ \\
\hline
\end{tabular}

paired proportions, and we assessed the effect using odds ratios and McNemar's test [20].

For statistical power, the power of McNemar's test is $73.5 \%$ for a small report (400 abstracts total) for which $10 \%$ of abstracts fell into the screening burden of Abstrackr but not EPPI-Reviewer, and another 5\% of abstracts fell into the screening burden of EPPI-Reviewer but not Abstrackr.

\section{Sensitivity at various thresholds}

Thresholds for screening truncation may be determined in several ways: screening may be stopped below a particular probability of inclusion or percentile rank or after consecutive exclusion of a large number of citations (e.g., inclusion rate $<0.1 \%$ in the last 500 screened). For each $10 \%$ set (i.e., $10 \%$, then $20 \%$, and then $30 \%$ ), we calculated the hypothetical sensitivity of the tool, which was the percentage of eventually included articles that had been screened by the tool at that point. This provided data on the time course of machine learning, which may vary by tool (e.g., Abstrackr or EPPI-Reviewer) or review (e.g., pancreatic cancer imaging or inguinal hernia treatment).

\section{Results}

\section{Included evidence reports and preparation of training} sets

Our databases contained 19,149 citations identified by searches for possible inclusion in these 9 reports. We 
excluded 505 duplicates to avoid delivering "mixed messages" for the machine learning algorithms (i.e., if a citation was included, but its duplicate was excluded for being a duplicate, this would confuse algorithms trained on abstract content alone). As we were unsure if either algorithm incorporated publication year into its prioritization scheme, we excluded 363 citations which had been excluded from the original pancreatic cancer imaging review solely for being published prior to 2000 . We included the remaining 18,281 articles.

Included reports covered a range of clinical topics, literature base sizes, and proportion of full-text orders and studies ultimately included (see Table 2). Although 10 articles appeared in searches for multiple topics, this did not impact our analytic comparisons, as citations from each report were entered as independent projects within each citation screening tool. In the two rightmost columns of Table 2, we provide the total number of articles that were ultimately ordered as full articles by the original reviewers (Task 1) as well as the total number of articles that were ultimately included in the review by the original reviewers (Task 2).

\section{Prioritization accuracy: EPPI-Reviewer and Abstrackr compared to traditional screening}

The impact of EPPI-Reviewer and Abstrackr on the amount of screening necessary for these tasks is shown in Figs. 3 and 4 below. For Task 1 (identifying citations for full-text review), both tools demonstrated potential for large reductions in screening for 2 of 3 large reports. In fact, for the indoor allergen reduction report, nearly all relevant citations were identified by the time $50 \%$ of citations had been screened (screening burden of $39.9 \%$ and $51.5 \%$ for EPPI-Reviewer and Abstrackr, respectively). Both tools also reduced screening for the third large report (surgical options for inguinal hernia) as well, but these reductions were far smaller $(<10 \%)$. Both tools were much less accurate in prioritization for smaller reports, particularly in Task 1, likely due to smaller training sets.

To place the results in Fig. 3 into context, we calculated the potential number of abstracts that users could be "saved" from having to screen by using each tool (Table 3). For example, the table shows that for the pancreatic cancer imaging report, Abstrackr could have "saved" 2612 abstracts from needing to be screened. (This is the $28.9 \%$ of abstracts that were prioritized lower than the last ordered article (100\% minus $71.1 \%$ that appears at the top of Fig. 3)). Overall, the numbers in Table 3 indicate substantial savings for the two largest reports, but modest savings for the other 7 reports.

As expected in Task 2 (training the tool using studies ultimately included), both tools identified included studies faster (i.e., earlier) compared to Task 1 (full-text-review decisions). For instance, for 2 of 3 large reports all studies included in the final report were identified by the time $41 \%$ of citations had been screened. Similar to Task 1 , both tools performed less well for the inguinal hernia report. Notably, both tools also performed well on the smaller reports, particularly EPPI-Reviewer, which reduced screening to $<60 \%$ for 4 of 6 small reports.

\section{Prioritization accuracy: EPPI-Reviewer vs. Abstrackr}

We compared performance of the two tools using McNemar's odds ratio. In Task 1, EPPI-Reviewer performed better than Abstrackr for all 3 large reports, consistently

Table 2 Citation disposition for 9 completed reports

\begin{tabular}{|c|c|c|c|c|}
\hline Report & $\begin{array}{l}\text { Citations for } \\
\text { screening }\end{array}$ & $\begin{array}{l}\text { Size of each } 10 \% \\
\text { training set }\end{array}$ & $\begin{array}{l}\text { Citations originally selected } \\
\text { for full text screening }(\boldsymbol{n}, \%)\end{array}$ & $\begin{array}{l}\text { Citations included } \\
\text { in final report } \\
(\boldsymbol{n}, \%)\end{array}$ \\
\hline \multicolumn{5}{|l|}{ Large reports } \\
\hline Pancreatic cancer imaging & 9038 & 904 & $696(8 \%)$ & $104(1 \%)$ \\
\hline Indoor allergen reduction & 3181 & 318 & $200(6 \%)$ & $72(2 \%)$ \\
\hline $\begin{array}{l}\text { Surgical procedures for } \\
\text { inguinal hernia }\end{array}$ & 2706 & 271 & $843(31 \%)$ & $223(8 \%)$ \\
\hline \multicolumn{5}{|l|}{ Small reports } \\
\hline Dabigatran & 889 & 89 & 107 (12\%) & $4(0.5 \%)$ \\
\hline $\begin{array}{l}\text { Transcatheter aortic valve } \\
\text { implantation (TAVI) }\end{array}$ & 673 & 67 & $267(40 \%)$ & $11(2 \%)$ \\
\hline Bronchial thermoplasty & 651 & 65 & $73(11 \%)$ & $15(2 \%)$ \\
\hline Digital tomosynthesis & 500 & 50 & $166(33 \%)$ & $12(2 \%)$ \\
\hline $\begin{array}{l}\text { Fecal transplantation for } \\
\text { Clostridium difficile }\end{array}$ & 427 & 43 & 149 (35\%) & $22(5 \%)$ \\
\hline Intragastric balloon & 226 & 23 & 104 (46\%) & $26(12 \%)$ \\
\hline
\end{tabular}

Counts in the "articles identified" column add to 10 more than the 18,281 article total because 10 articles had been identified for possible inclusion in 2 projects 


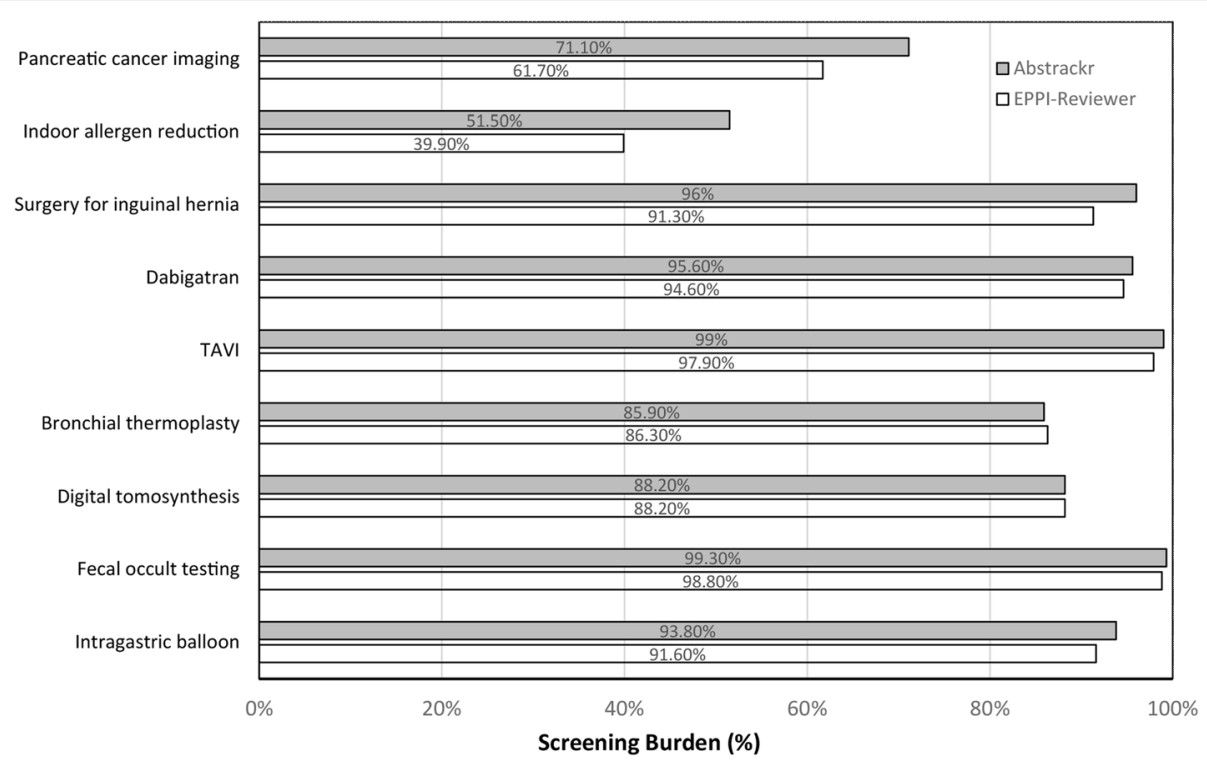

Fig. 3 Prioritization accuracy for Task 1 (inclusion for full-text screening). This figure shows the percentage of articles screened by each tool to reach $100 \%$ of all articles ordered for full-text screening. Smaller bars indicate higher prioritization accuracy (as fewer articles had to be screened in order to achieve 100\% sensitivity). The comparative performance of the two tools for each evidence report is displayed in gray (Abstrackr) and white (EPPI-Reviewer)

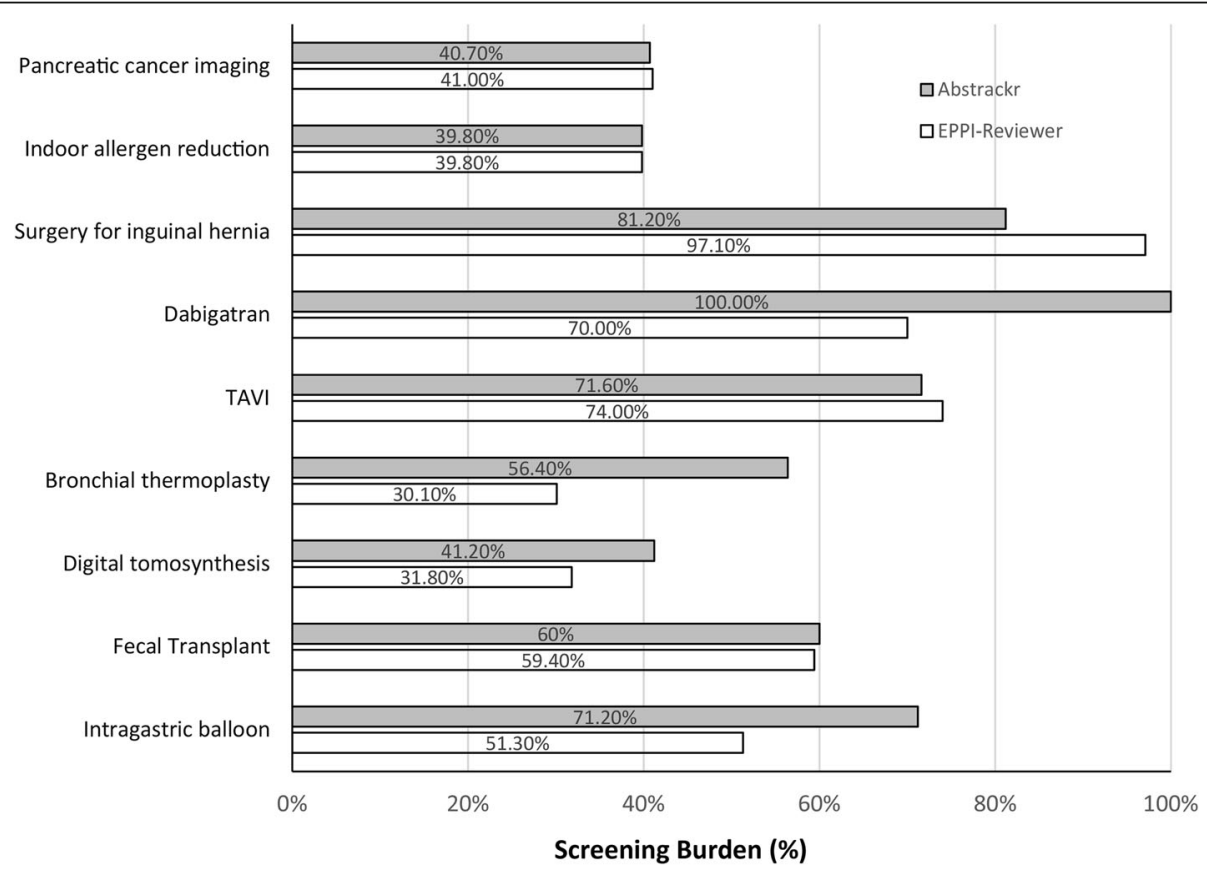

Fig. 4 Prioritization accuracy for Task 2 (final inclusion). This figure shows the percentage of articles screened by each tool to reach $100 \%$ of all articles included in the final report. Smaller bars indicate higher prioritization accuracy (as fewer articles had to be screened in order to achieve $100 \%$ sensitivity). The comparative performance of the two tools for each evidence report is displayed in gray (Abstrackr) and white (EPPI-Reviewer) 
Table 3 Abstract screenings saved

\begin{tabular}{|c|c|c|}
\hline Report & $\begin{array}{l}\text { Number of abstract } \\
\text { screenings potentially } \\
\text { saved by Abstrackr }\end{array}$ & $\begin{array}{l}\text { Number of abstract } \\
\text { screenings potentially } \\
\text { saved by EPPI-Reviewer }\end{array}$ \\
\hline \multicolumn{3}{|l|}{ Large reports } \\
\hline $\begin{array}{l}\text { Pancreatic cancer } \\
\text { imaging }(N=9038)\end{array}$ & 2612 & 3462 \\
\hline $\begin{array}{l}\text { Indoor allergen } \\
\text { reduction }(N=3181)\end{array}$ & 1540 & 1912 \\
\hline $\begin{array}{l}\text { Surgical procedures } \\
\text { for inguinal hernia } \\
(N=2706)\end{array}$ & 108 & 235 \\
\hline \multicolumn{3}{|l|}{ Small reports } \\
\hline Dabigatran $(N=889)$ & 39 & 48 \\
\hline $\begin{array}{l}\text { Transcatheter aortic } \\
\text { valve implantation } \\
\text { (TAVI) }(N=673)\end{array}$ & 7 & 14 \\
\hline $\begin{array}{l}\text { Bronchial } \\
\text { thermoplasty } \\
(N=651)\end{array}$ & 92 & 89 \\
\hline $\begin{array}{l}\text { Digital tomosynthesis } \\
(N=500)\end{array}$ & 59 & 59 \\
\hline $\begin{array}{l}\text { Fecal transplantation } \\
\text { for Clostridium difficile } \\
(N=427)\end{array}$ & 3 & 5 \\
\hline $\begin{array}{l}\text { Intragastric balloon } \\
(N=226)\end{array}$ & 14 & 19 \\
\hline
\end{tabular}

identifying relevant studies for full text ordering faster than Abstrackr (see Fig. 5, where for the top 3 datapoints, confidence intervals are fully to the left of a null effect). For example, for Task 1 for pancreatic cancer imaging, EPPI-Reviewer demonstrated a potential reduction in screening burden of nearly 10\% compared to Abstrackr, identifying all abstracts ordered for full text 868 citations earlier. For indoor allergens and surgical interventions for inguinal hernia, EPPI-Reviewer provided reductions of $12 \%$ and 5\% translating to 369 and 127 citations, respectively. EPPI-Reviewer also appeared to perform better for several of the smaller reports, although those results did not reach statistical significance.

However, for Task 2 (final inclusion predictions) results were mixed: Abstrackr performed better for reviews on indoor allergens and surgical interventions for inguinal hernia, but EPPI-Reviewer performed better for dabigatran, bronchial thermoplasty, digital tomosynthesis, and intragastric balloon (all statistically significant).

\section{Sensitivity at progressive screening thresholds}

We also assessed how many relevant citations had been identified at successive $10 \%$ intervals for each screening tool. Data for the 3 large reports are shown in Fig. 6 (pancreatic cancer), Fig. 7 (indoor allergens), and Fig. 8 (inguinal hernia) (we did not produce these graphs for the small reports, due to small Ns). In these figures, the horizontal axis represents the percentage of all articles screened (in 10\% increments), while the vertical axis plots the sensitivity (defined as the percentage of included articles screened at that point).

As each tool was trained with $10 \%$ of citations and Abstrackr only updates daily, at $10 \%$ of citations screened, EPPI-Reviewer consistently outperformed Abstrackr. This was expected, as Abstrackr did not have the opportunity to re-order citations until 10\% had been screened; thus, the order at $10 \%$ screened simply reflects a random $10 \%$ sample. By contrast, for EPPI-Reviewer, after 10 random citations were screened as "training," we engaged the screening prioritization functionality, allowing EPPIReviewer to iteratively reorder citations as soon as sufficient data for prediction was acquired. EPPI-Reviewer's edge over Abstrackr at 10\% of citations screened was expected, but nevertheless demonstrates the pragmatic advantages to EPPI-Reviewer's iterative approach which allows identifying relevant articles earlier.

Both tools learned quickly for 2 of 3 large reviews (pancreatic cancer imaging and indoor allergen reduction). For pancreatic cancer, at only $30 \%$ citations screened, both tools had already identified $\geq 85 \%$ of all relevant studies in both tasks. By $40 \%$ screening, both tools had screened $98.1 \%$ of studies included in the final report (Task 2). For indoor allergen reduction, performance was even more impressive: at only 30\% screening, Abstrackr had screened $98.6 \%$ of the final included studies, and EPPI-Reviewer had screened 97.2\%. Perfect sensitivity $(100 \%)$ was achieved by screening only $31.8 \%$ of citations (Abstrackr) and 39.9\% (EPPI-Reviewer) (Fig. 9).

Performance was poorer for the third large review, inguinal hernia (Figs. 10 and 11). Although a significant proportion of citations included in the report were reached by $30 \%$ of citations screened (83\% for Abstrackr, $86 \%$ for EPPI-Reviewer), very high sensitivity (99\% for Abstrackr, 98\% for EPPI-Reviewer) was not reached until $70 \%$ of citations had been screened. For both tools, 21 relevant citations were not screened until the bottom $50 \%$ of all articles. Thus, although performing substantively better than chance, both tools performed less well for this project compared to the other 2 large projects.

\section{Discussion}

Our study found both Abstrackr and EPPI-Reviewer have the potential to reduce screening burden. Specifically, for large reports, potential reductions in screening burden were 4 to 49\% (Abstrackr) and 9 to 60\% (EPPIReviewer). Our findings are consistent with other studies which have found Abstrackr to offer potential gains [7, 19, 21]. However, to our knowledge, our study is the first to assess EPPI-Reviewer. Overall, compared to Abstrackr, we found that EPPI-Reviewer offered similar 


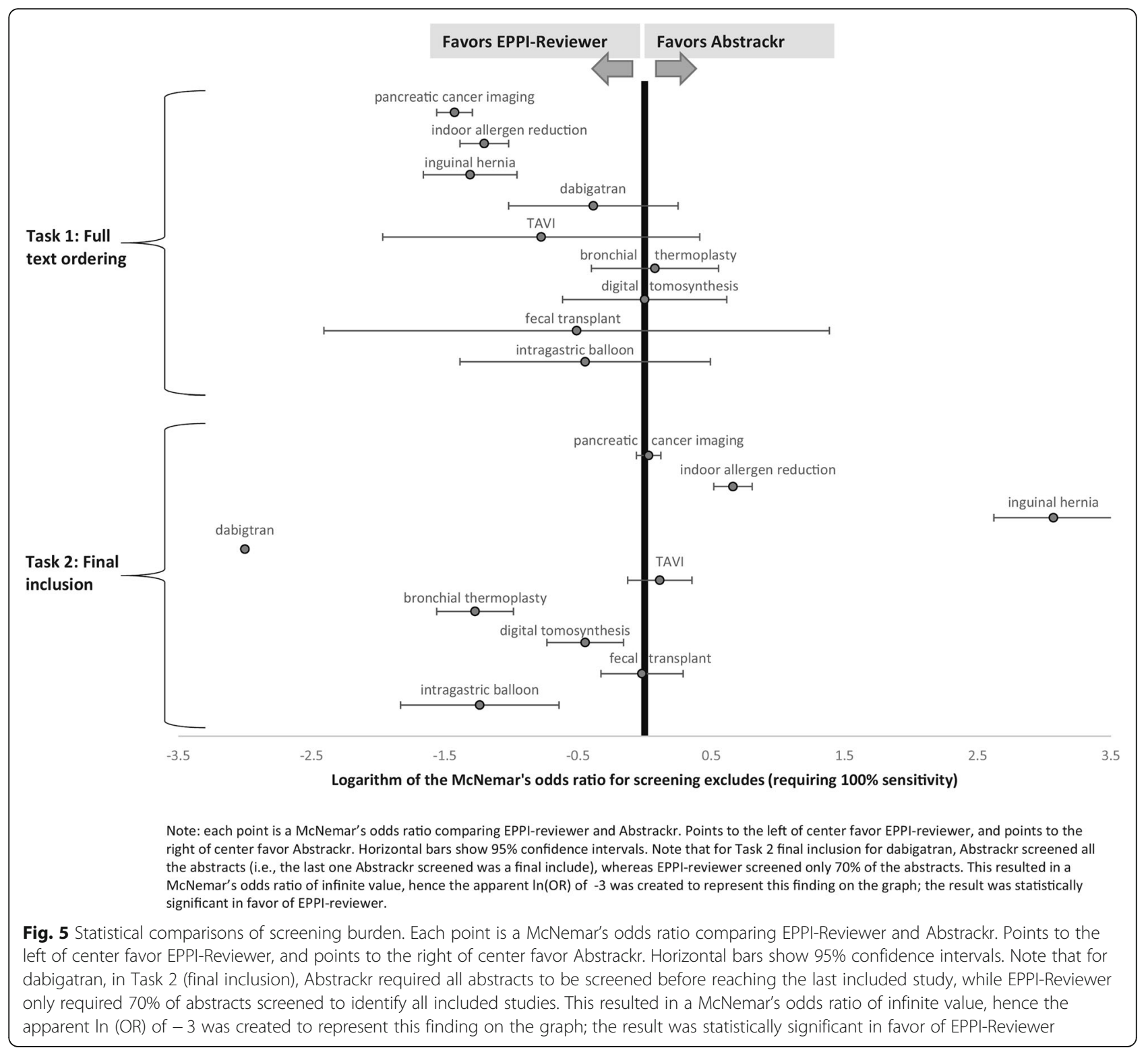

prioritization accuracy. In 2 of 3 large evidence reports, both tools performed well for both Tasks 1 and 2 .

Performance for the third large report (surgical interventions for inguinal hernia) was less impressive for both tools: in Task 1, 91.3\% and 96\% of citations required screening (for EPPI-Reviewer and Abstrackr respectively) before all citations ordered for full text were screened. One possibility for this poorer performance could be that citations not identified until late in the screening process represent studies that were somewhat "off topic, " but were included for further review as part of a cautious approach. In this scenario, one might expect many of these studies to ultimately be excluded from the final report. In this case, the prediction algorithm might be unfairly penalized for designating these citations low priority for full text ordering (e.g., correctly assessing the potential for inclusion as very low). However, our review of studies identified very late for Task 1 (full text ordering) suggests some citations falling in this "tail" were ultimately included in the final report. After $70 \%$ of citations had been screened, both citation tools had not yet identified 4 (of 223 total) studies that would be included in the final report.

These studies identified late in screening may have been harder for Abstrackr/EPPI-Reviewer to identify because they differed in key ways from studies identified earlier in the process. Of 223 studies included, 62 (reporting 32 unique studies) addressed a key question on the learning curve for laparoscopic hernia repair. Inclusion criteria for this key question differed from other questions in the report; specifically, many single-arm studies excluded from consideration for other key questions were allowed for this 


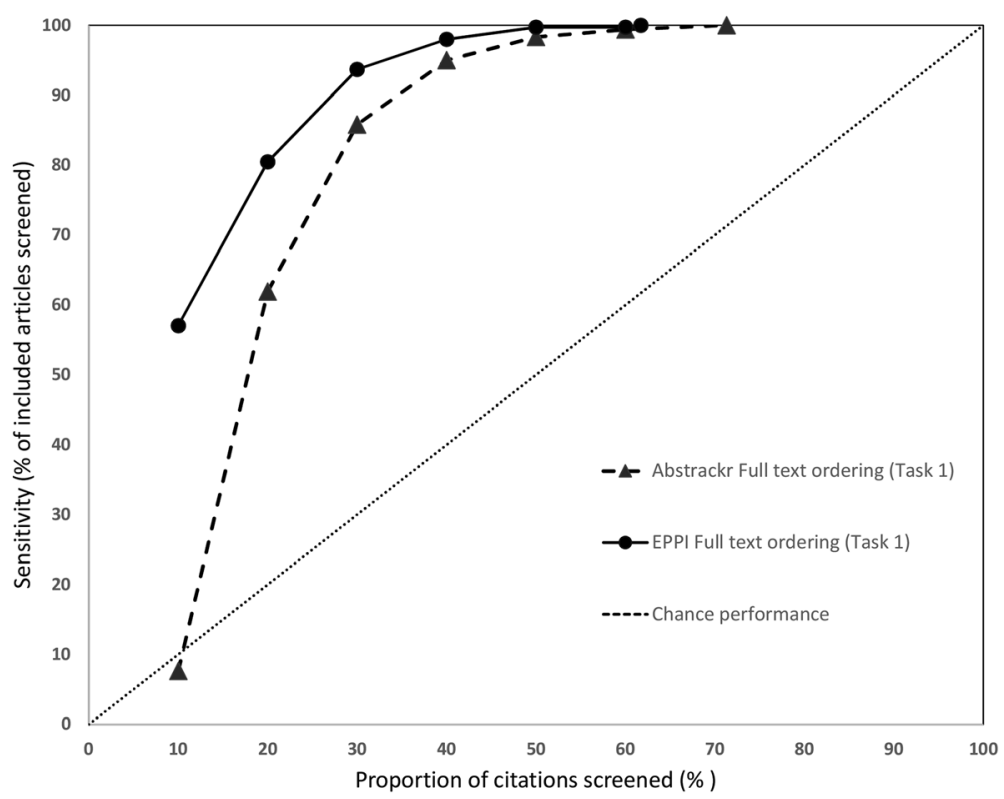

Fig. 6 Sensitivity at various thresholds for pancreatic cancer imaging (Task 1). This figure shows the proportion of included articles that were screened at each 10\% increment. Performance for both Abstrackr (dotted lines with triangles) and EPPI-Reviewer (solid lines with circles) is plotted. Chance performance is shown by the 45 degree line. As the $y$-axis plots sensitivity, curves closer to the top left of the graph indicate faster learning

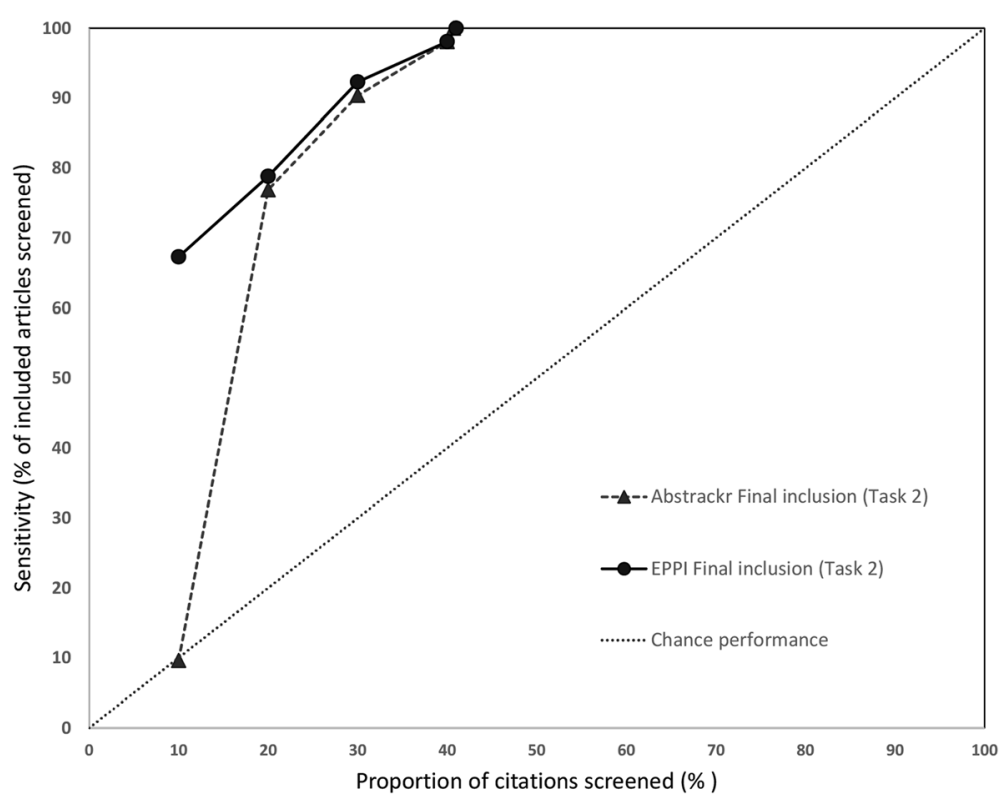

Fig. 7 Sensivity at various thresholds for pancreatic cancer imaging (Task 2). This figure shows the proportion of included articles that were screened at each 10\% increment. Performance for both Abstrackr (dotted lines with triangles) and EPPI-Reviewer (solid lines with circles) is plotted. Chance performance is shown by the 45 degree line. As the $y$-axis plots sensitivity, curves closer to the top left of the graph indicate faster learning 


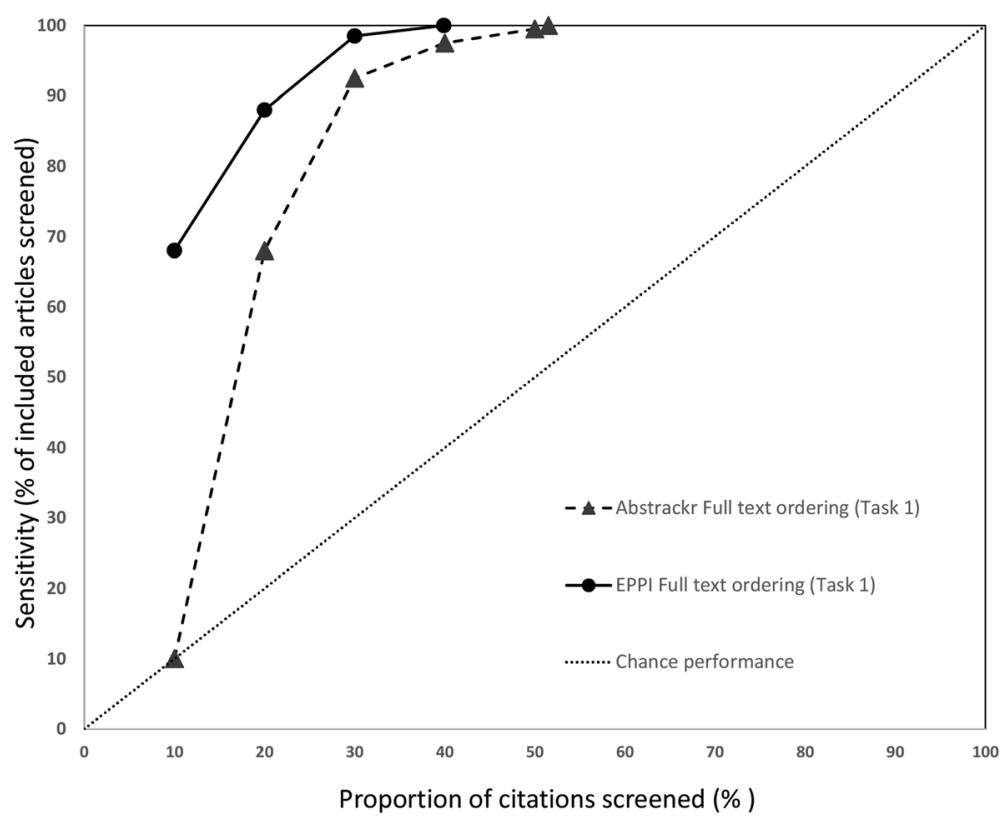

Fig. 8 Sensitivity at various thresholds for indoor allergen reduction (Task 1). This figure shows the proportion of included articles that were screened at each 10\% increment. Performance for both Abstrackr (dotted lines with triangles) and EPPI-Reviewer (solid lines with circles) is plotted. Chance performance is shown by the 45 degree line. As the $y$-axis plots sensitivity, curves closer to the top left of the graph indicate faster learning

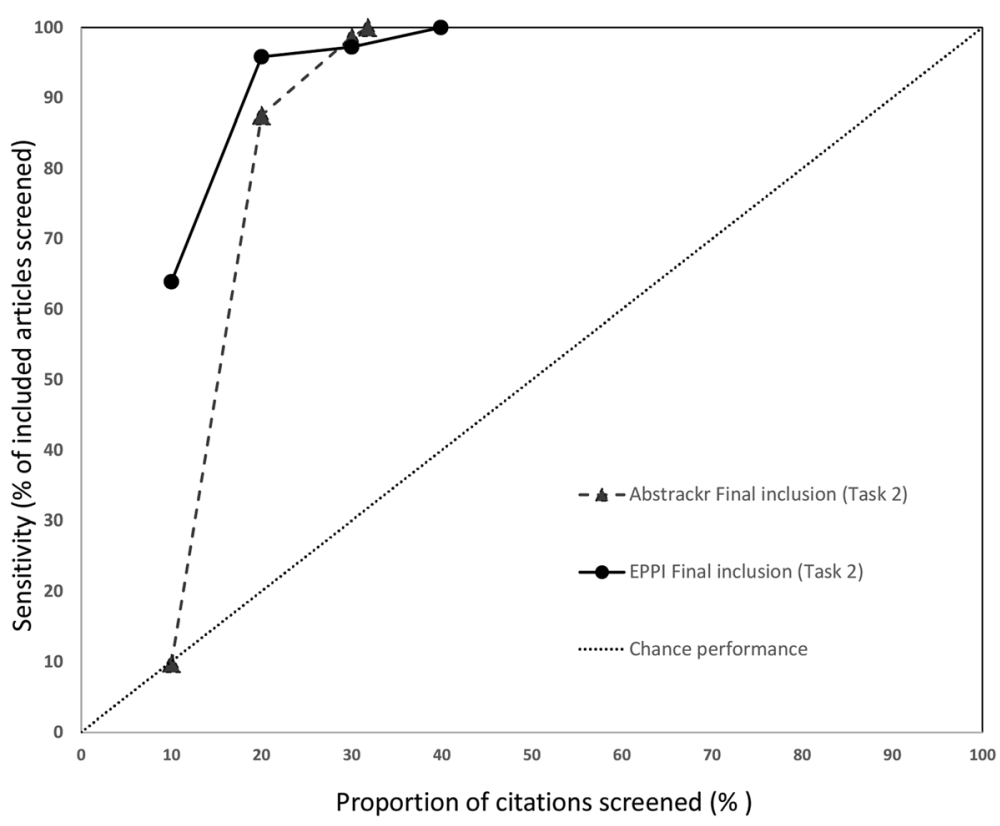

Fig. 9 Sensitivy at various thresholds for indoor allergen reduction (Task 2). This figure shows the proportion of included articles that were screened at each 10\% increment. Performance for both Abstrackr (dotted lines with triangles) and EPPI-Reviewer (solid lines with circles) is plotted. Chance performance is shown by the 45 degree line. As the $y$-axis plots sensitivity, curves closer to the top left of the graph indicate faster learning 


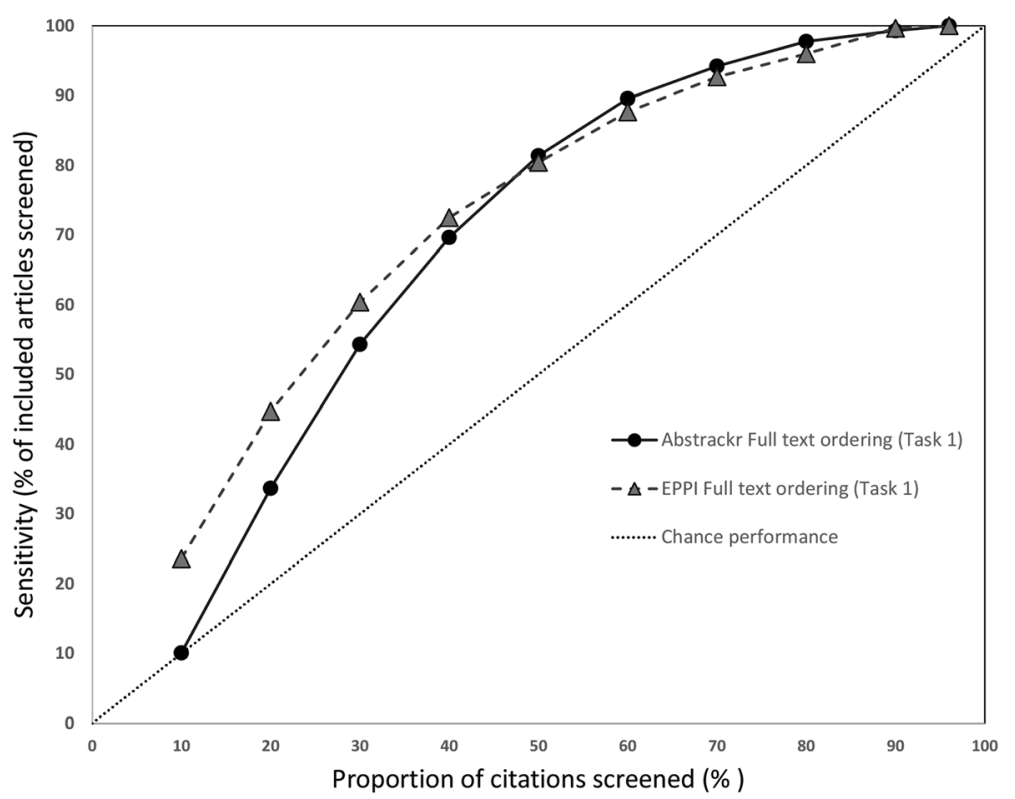

Fig. 10 Sensitivity at various thresholds, surgical interventions for inguinal hernia (Task 1). This figure shows the proportion of included articles that were screened at each 10\% increment. Performance for both Abstrackr (dotted lines with triangles) and EPPI-Reviewer (solid lines with circles) is plotted. Chance performance is shown by the 45 degree line. As the $y$-axis plots sensitivity, curves closer to the top left of the graph indicate faster learning

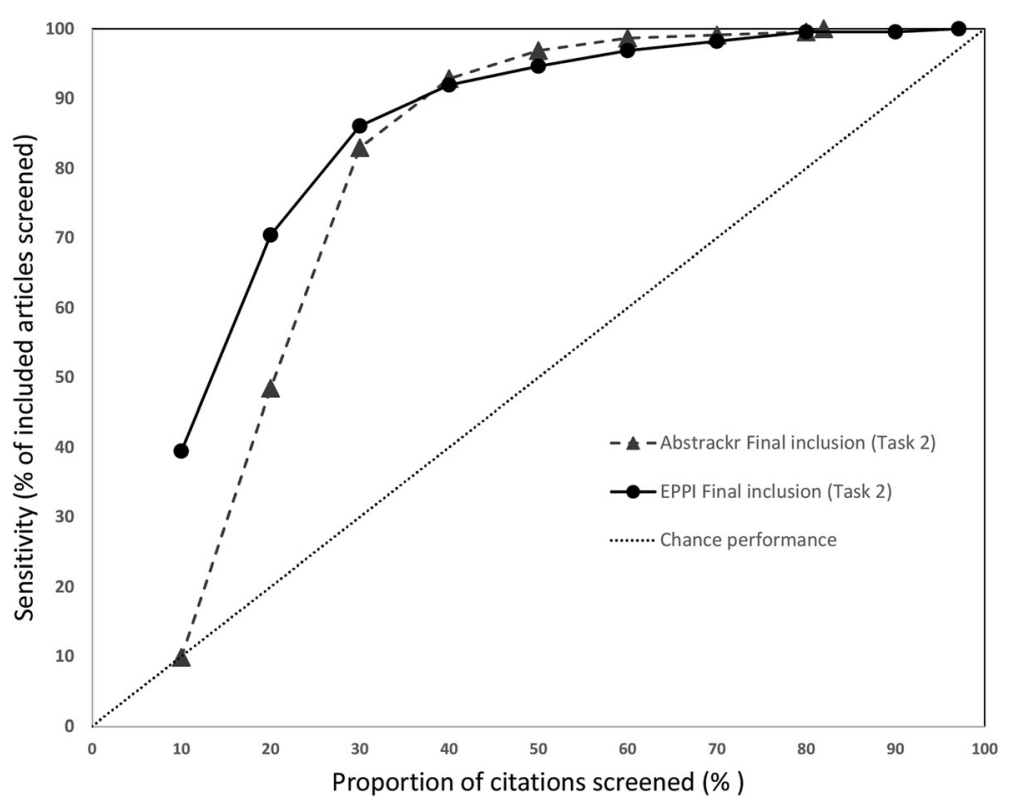

Fig. 11 Sensivity at various thresholds, surgical interventions for inguinal hernia (Task 2). This figure shows the proportion of included articles that were screened at each 10\% increment. Performance for both Abstrackr (dotted lines with triangles) and EPPI-Reviewer (solid lines with circles) is plotted. Chance performance is shown by the 45 degree line. As the $y$-axis plots sensitivity, curves closer to the top left of the graph indicate faster learning 
key question. Of 62 publications included for this key question, 57 were in the bottom $50 \%$ of priority for either Abstrackr or EPPI-Reviewer (or both). This suggests the other 161 included publications, many of which described randomized trials, may have influenced algorithms to prioritize only randomized trials.

If machine learning algorithms are focused on clinical content or study design, studies included or excluded based on a non-clinical study characteristic (e.g., publication date or study size) would likely reduce predictive power. One potential strategy for minimizing the impact of such factors in the future would be to isolate key questions with distinctive populations or inclusion criteria by separating them from other key questions (i.e., training algorithms for these questions separately).

Interestingly, the original search for surgical interventions for inguinal hernia was distinctly more challenging, perhaps due to the fact that it is a procedure-based topic not well-indexed with controlled vocabulary terms. It is possible that the same factors creating challenges for crafting a search with adequate sensitivity and specificity could also pose problems for machine learning algorithms.

\section{Small reports}

For smaller reports, neither tool offered significant gains for Task 1 (predicting full text ordering). However, for Task 2 (which trained tools using only articles included in the final report) both tools performed surprisingly well, particularly EPPI-Reviewer. In 4 of 6 small reports, EPPI reviewer identified all included citations earlier than Abstrackr, offering reductions in screening burden of 30 to $70 \%$ (see Fig. 5). This suggests that even for small reports, tools like EPPI-Reviewer can offer efficiency gains, particularly for playing a confirmatory role (e.g., checking for missed citations at the end of a review) or updating reviews, both situations in which a high-quality training set is available.

\section{Encouraging wider adoption}

Despite numerous tools for automation, adoption of these tools has lagged $[8,22]$. Reasons for slower uptake may include pragmatic challenges from incorporating tools into current workflow, and lack of trust by the systematic review community [22]. Many studies evaluating these tools have assessed automatic classification functionality, in which screening continued until the tool predicts whether studies should be included or not, and remaining citations are not screened $[19,21]$. However, existing uncertainties regarding how to ensure tools like Abstrackr and EPPI-Reviewer perform at their best suggest that perhaps utilizing these tools for screening prioritization remains the safest choice for those concerned about missing any relevant citations. If a "trust gap" is impeding adoption, perhaps encouraging initial use of these tools for screening prioritization, while accumulating new evidence for specific contexts in which automatic classification may safely be used is the way forward.

\section{Special considerations for use}

Using screening prioritization tools may require careful consideration of factors which could potentially reduce predictive performance. For instance, as previously noted, we carefully removed all duplicates from the citation set. If one study is included, but a second study is excluded as duplicate (i.e., not due to irrelevant content), this could potentially confuse an algorithm learning on content alone. Similarly, excluding studies based on non-clinical characteristics such as publication year, country, or sample size could similarly confuse the prediction algorithm. For example, if a study evaluated an intervention of interest, but was excluded solely based on small sample size, the predictive algorithm might mistakenly conclude the intervention was irrelevant.

Secondary publications may also pose a challenge: if one study population appears in multiple publications, authors may elect to include the "primary" original study, while excluding secondary publications (additional publications from the same study). Reviewers would need to be cognizant of the possibility of sending mixed messages to the machine learning algorithm. To maximize benefits from these tools, reviewers would either need to specify inclusion of all secondary publications or "clean" the citation set prior to training the citation screening tool.

\section{Limitations}

Our study has important limitations. Performance of Abstrackr and EPPI-Reviewer may significantly vary depending on composition of the initial training set. We trained Abstrackr using a random $10 \%$ of citations. If studies in this training set were not representative, the predictive algorithm's "first impressions" may have been skewed, affecting performance (e.g., repeating the study with a different random $10 \%$ training set could have produced different results). Similarly, we used a random 10 citations to train EPPI-Reviewer; using a different 10 studies could produce different results. Also, notably, both tools offer features which could improve performance in "real life" use (e.g., identification of key words and automatic classification) which we chose not use, in order to facilitate comparison across tools.

Similarly, we assessed performance at intervals of $10 \%$ citations screened rather than counts of numbers of studies (e.g., every 100 citations). We chose 10\% intervals to capture incremental improvement with progression of screening, but also to accommodate pragmatic considerations. For instance, as Abstrackr updates the order of citations only once a day, to complete screening of $>9000$ 
citations for the pancreatic cancer imaging report in 100 citation increments would have required 90 days. However, we recognize a $10 \%$ increment translates to significant differences in actual number of citations screened across reports (e.g., 904 citations for the pancreatic imaging report compared to only 271 citations for inguinal hernia) and results may have differed had we analyzed results by number of citations screened alone.

Despite the potential for significant reductions in screening burden (particularly for large reports with high numbers of citations) a fundamental barrier to wider uptake is the inability to know in advance whether tool performance is excellent (e.g., pancreatic cancer imaging and indoor allergens) or less good (e.g., inguinal hernia). For example, for the pancreatic imaging report, EPPIReviewer identified all citations ordered for full-text review after only $62 \%$ of citations had been screened. However, in "real life" use, systematic reviewers cannot simply stop screening at this point (to avoid screening the remaining 3400 citations). Prospectively, it remains impossible to discern whether the predictive performance will be "good" or "bad." Thus, our data on "potential" reductions in screening burden may not translate into actual reductions for real-time use.

Nevertheless, our findings suggest that a conservative approach could expect to identify nearly all citations for inclusion after screening $70-80 \%$ of citations using these tools. A more daring approach would be to stop screening after $50 \%$ of citations have been screened, which our study suggests would still identify roughly $95 \%$ of included studies. More work is needed to evaluate the tradeoffs associated with truncating screening early (e.g., at 50\%) to assess to what extent these "missed" studies impact the report's conclusions. Although this technology is in the early stages of adoption, as these tools become more commonplace and integrated into routine SR development, some of these factors may change. Training sets based on initial versions of SRs could facilitate highly desirable living SRs where new citations are considered for relevancy in a predetermined ongoing process. Libraries of training sets (along with key questions they were designed to answer) potentially could streamline prioritization for related new reviews.

\section{Conclusions}

Both EPPI-Reviewer and Abstrackr performed well for predicting relevant citations, both for full-text review, and for final inclusion. Our findings suggest these tools are safe for use in confirmatory roles (e.g., to double-check if relevant citations have been missed) or for use in ongoing systematic reviews, as many have suggested. As expected, for new review development, these tools were most beneficial for larger citation sets (with $\geq 2500$ citations). While these tools can expedite workflow efficiency by identifying relevant citations earlier, more work is needed to determine the impact of studies that may be missed if screening is truncated below a particular threshold. Future research should also assess the risks and tradeoffs associated with using these tools prospectively.

\section{Significance}

Data from this project may inform decisions on how to shorten the article screening process. Such decisions might arise in the context of a rapid review, a review update, or general efficiency gains.

\section{Acknowledgements}

We gratefully acknowledge the invaluable assistance of Helen Dunn, Nancy Sullivan, Linnea Hermanson, Amber Moran, and Kariann Hudson in retrieving citation datasets (HD), and screening citations (NS, LH, AM, KH). We also thank Jeff Brunton from EPPI-Reviewer for downloading EPPI-Reviewer data for us repeatedly throughout the project. Finally, we thank Lionel Banez, M.D. (Agency for Healthcare Research and Quality) for providing support and feedback.

\section{Authors' contributions}

AT and JT contributed to the conception, design, acquisition of data, analysis, interpretation of data, drafting and revision of the manuscript; $\mathrm{EE}$ contributed to the conception, design, and revision of the manuscript; KS contributed to the design and revision of the manuscript. All authors have approved the submitted version of this manuscript and agree to be personally accountable for the author's own contributions as well as parts of the work in which the author was not personally involved.

\section{Funding}

This work was funded by the Agency for Healthcare Research and Quality (AHRQ) Grant Number R03 HS25859.

\section{Availability of data and materials}

The datasets generated and analyzed during the current study are not publicly available as other research projects are ongoing but are available from the corresponding author on reasonable request.

Ethics approval and consent to participate

Not applicable.

Consent for publication

Not applicable

\section{Competing interests}

The authors declare that they have no competing interests.

Received: 19 November 2019 Accepted: 8 March 2020

Published online: 02 April 2020

\section{References}

1. Sackett, David R. Evidence-based medicine: how to practice and teach EBM, 2nd Edition: By David L. Sackett, Sharon E. Straus, W. Scott Richardson, William Rosenberg, and R. Brian Haynes, Churchill Livingstone, 2000. Vol. 16. 2001 [cited 2019 Jul 18]. Available from: https://doi.org/10.1177/ 088506660101600307.

2. Committee on Standards for Systematic Reviews, Institute of Medicine. Finding what works in health care: standards for systematic reviews. 2011 [cited 2019 Jul 18]. Available from: https://www.nap.edu/catalog/13059/ finding-what-works-in-health-care-standards-for-systematic-reviews.

3. Institute of Medicine (US) Committee on Standards for Developing Trustworthy Clinical Practice Guidelines. In: Graham R, Mancher M, Miller Wolman D, Greenfield S, Steinberg E, editors. Clinical practice guidelines we can trust. Washington: National Academies Press (US); 2011. [cited 2019 Nov 14]. Available from: http://www.ncbi.n/m.nih.gov/books/NBK209539/.

4. Shekelle PG. Clinical practice guidelines: what's next? JAMA. 2018;320(8): 757-8. 
5. Borah R, Brown AW, Capers PL, Kaiser KA. Analysis of the time and workers needed to conduct systematic reviews of medical interventions using data from the PROSPERO registry. BMJ Open. 2017;7(2):e012545.

6. Tricco AC, Brehaut J, Chen MH, Moher D. Following 411 Cochrane protocols to completion: a retrospective cohort study. PLoS One. 2008;3(11):e3684.

7. Wallace BC, Trikalinos TA, Lau J, Brodley C, Schmid CH. Semi-automated screening of biomedical citations for systematic reviews. BMC Bioinformatics. 2010;11:55.

8. Lau J. Editorial: systematic review automation thematic series. Syst Rev. 2019;8(1):70.

9. Harrison H, Griffin SJ, Kuhn I, Usher-Smith JA. Software tools to support title and abstract screening for systematic reviews in healthcare: an evaluation. BMC Med Res Methodol. 2020;20(1):7.

10. O'Mara-Eves A, Thomas J, McNaught J, Miwa M, Ananiadou S. Using text mining for study identification in systematic reviews: a systematic review of current approaches. Syst Rev. 2015;4:5.

11. Przybyła P, Brockmeier AJ, Kontonatsios G, Le Pogam M, McNaught J, von Elm E, et al. Prioritising references for systematic reviews with RobotAnalyst: a user study. Res Synth Methods. 2018;9(3):470-88.

12. Paynter R, Bañez LL, Erinoff E, Lege-Matsuura J, Potter S. Commentary on EPC methods: an exploration of the use of text-mining software in systematic reviews. J Clin Epidemiol. 2017;84:33-6.

13. Shemilt I, Simon A, Hollands GJ, Marteau TM, Ogilvie D, O'Mara-Eves A, et al. Pinpointing needles in giant haystacks: use of text mining to reduce impractical screening workload in extremely large scoping reviews. Res Synth Methods. 2014:5(1):31-49.

14. Thomas J. Text mining for reducing screening workload: is it safe to use? [cited 2019 Jul 19]. Available from: https:/methods.cochrane.org/sites/ default/files/public/uploads/james\%20thomas.pdf.

15. Paynter R, Bañez LL, Berliner E, Erinoff E, Lege-Matsuura J, Potter S, et al. EPC methods: an exploration of the use of text-mining software in systematic reviews. Rockville: Agency for Healthcare Research and Quality (US); 2016. [cited 2019 Nov 8]. (AHRQ Methods for Effective Health Care). Available from: http://www.ncbi.nlm.nih.gov/books/NBK362044/.

16. abstrackr: home. [cited $2019 \mathrm{Jul}$ 18]. Available from: http://abstrackr.cebm. brown.edu/account/login.

17. Wallace BC, Small K, Brodley CE, Lau J, Trikalinos TA. Deploying an interactive machine learning system in an evidence-based practice center: abstrackr. In: Proceedings of the 2nd ACM SIGHIT symposium on International health informatics - IHI '12. Miami, Florida, USA: ACM Press; 2012. [cited 2019 Jul 18]. p. 819. Available from: http://dl.acm.org/citation. cfm?doid=2110363.2110464.

18. EPPI-Reviewer Web (Beta) [Internet]. [cited 2019 Jul 18]. Available from: https://eppi.ioe.ac.uk/eppireviewer-web/home.

19. Gates A, Johnson C, Hartling L. Technology-assisted title and abstract screening for systematic reviews: a retrospective evaluation of the Abstrackr machine learning tool. Syst Rev. 2018;7 [cited 2019 Oct 10] Available from: https://www.ncbi.nlm.nih.gov/pmc/articles/PMC5848519/.

20. Armitage P. Statistical methods in medical research. 3rd ed. Oxford, England: Blackwell Scientific; 1994. p. 826.

21. Rathbone J, Hoffmann T, Glasziou P. Faster title and abstract screening? Evaluating Abstrackr, a semi-automated online screening program for systematic reviewers. Syst Rev. 2015;4:80.

22. O'Connor AM, Tsafnat G, Thomas J, Glasziou P, Gilbert SB, Hutton B. A question of trust: can we build an evidence base to gain trust in systematic review automation technologies? Syst Rev. 2019;8(1):143.

\section{Publisher's Note}

Springer Nature remains neutral with regard to jurisdictional claims in published maps and institutional affiliations.

Ready to submit your research? Choose BMC and benefit from:

- fast, convenient online submission

- thorough peer review by experienced researchers in your field

- rapid publication on acceptance

- support for research data, including large and complex data types

- gold Open Access which fosters wider collaboration and increased citations

- maximum visibility for your research: over $100 \mathrm{M}$ website views per year

At BMC, research is always in progress.

Learn more biomedcentral.com/submissions 